\title{
How and why a bit-wise neutrality with and without locality affects evolutionary search
}

\author{
Edgar Galvan-Lopez \\ Department of Computer Science \\ University of Essex, UK \\ egalva@essex.ac.uk
}

\author{
Riccardo Poli \\ Department of Computer Science \\ University of Essex, UK \\ rpoli@essex.ac.uk
}

\begin{abstract}
Despite the vast work on neutrality, there are not general conclusions on its effects. In this paper we make an effort to understand how neutrality influences evolution. For this purpose we will use a type of neutrality that allows locality (which is believed to be a desirable feature of neutrality).

Categories and Subject Descriptors: I.2.8 Artificial Intelligence [Problem Solving, Control Methods and Search]: Heuristic methods
\end{abstract}

General Terms: Algorithms

Keywords: Genetic Algorithms, Neutrality, Locality

\section{INTRODUCTION}

Neutral theory of molecular evolution was proposed by Kimura [2] in the late 1960s. Kimura stated that when one compares the genomes of species, the majority of molecular differences are neutral. In other words, these molecular changes represented by these differences do not influence the fitness of the individual. The results reported when neutrality is added to the evolutionary process are contradictory and so, there are no general conclusions on its effects.

\section{PROPOSED APPROACH AND RESULTS}

In previous investigations $[1,3]$, we have started sheding some light on neutrality. In this paper, we intend to further our understanding of when neutrality may be beneficial. To do so: (a) we use two problems with significantly different landscape features: a unimodal landscape and a multimodal deceptive landscape, (b) we used a mutation based, binaryGA without crossover and which is modified in such a way to allow locality ${ }^{1}$.

Once we have create our initial population, we modified it in the following way: (a) we find those individuals (which we call original points) that have more than one neighbour in the population (Hamming distance 1 away). Neighbours are assigned the fitness of the corresponding original points, (b) if an individual has two original points, then such individual will take the fitness of the original point that has fewer neighbours. In case there is a tie, then the fitness of the individual will be calculated as usual, (c) if an individual

${ }^{1}$ Creating neutral networks where individuals are neighbours in the Hamming space (Hamming distance $=1$ ).

Copyright is held by the author/owner(s).

GECCO'07, July 7-11, 2007, London, England, United Kingdom.

ACM 978-1-59593-697-4/07/0007.
Table 1: Results found on the OneMax problem and the Trap function when neutrality is added.

\begin{tabular}{|c|cc|cc|}
\hline & \multicolumn{2}{|c|}{ OneMax } & \multicolumn{2}{c|}{ Trap Function } \\
& With & Without & With & Without \\
& Neutral & Neutral & Neutral & Neutral \\
\hline \hline$P_{\text {mut }}=0.01$ & $2.7 \%$ & $100 \%$ & $1.1 \%$ & $0.3 \%$ \\
$P_{\text {mut }}=0.06$ & $15.1 \%$ & $100 \%$ & $10.3 \%$ & $0.7 \%$ \\
$P_{\text {mut }}=0.1$ & $17.7 \%$ & $100 \%$ & $15.0 \%$ & $0.7 \%$ \\
\hline
\end{tabular}

has more than two original points, then the individual will not be part of any neutral network and will keep its fitness.

To test our approach, we have used: population size $=80$, generations $=100$, mutation rates (per bit) $=\{0.01,0.06$, $0.1\}$ and 1,000 independent runs. For the Trap function we have used the function:

$$
f(X)= \begin{cases}\frac{a}{z}(z-u(X)) & \text { if } u(X) \leq z \\ \frac{b}{k-z}(u(X)-z) & \text { otherwise }\end{cases}
$$

where $a$ is the deceptive optimum, $b$ is the global optimum, and $z$ is the slope-change location. For this problem we used $k=14, z=13, a=39, b=40$ (with these parameters, the problem is very difficult). In Table 1 , we show the results.

\section{CONCLUSIONS}

We argue that reasons of why neutrality is a controversial area derive from the fact that different researchers use radically different types of neutrality on problems with different landscape feautures and rarely an effort has been made to understand exactly how the search space has been modified by the presence of neutrality.

In this paper we have made an effort to understand the effects of neutrality in the presence of locality. We argue that neutrality may be beneficial in certain circumstances and to illustrate this we have used two problems with very different landscape features.

\section{REFERENCES}

[1] E. Galván-López, and R. Poli, An Empirical Investigation of How and Why Neutrality Affects Evolutionary Search. In GECCO 2006, pages 1149-1156, 2006, ACM Press.

[2] M. Kimura, Evolutionary rate at the molecular level. In Nature, volume 217, pages 624-626, 1968.

[3] R. Poli and E. Galván-López, On the effects of bit-wise neutrality on fitness distance correlation, phenotypic mutation rates and problem hardness. FOGA IX, 8-11, Jan., 2007, Springer-Verlag. 